\title{
El nivel de vocabulario como indicador de reserva cognitiva en la evaluación del deterioro cognitivo ligero
}

\author{
Cristina Lojo-Seoane*, David Facal, Onésimo Juncos-Rabadán y Arturo X. Pereiro
}

Universidade de Santiago de Compostela, Departamento de Psicoloxía evolutiva y da Educación

\begin{abstract}
Resumen: El presente trabajo estudia el papel del vocabulario, como indicador de reserva cognitiva, en la evaluación del Deterioro Cognitivo Ligero (DCL). Participaron 326 adultos mayores de 50 años, clasificados en dos grupos, uno de 104 participantes con DCL y otro de 222 controles sanos. Se analizaron las diferencias en las puntuaciones obtenidas en distintos indicadores de reserva cognitiva, entre ellos, las puntuaciones en el subtest de vocabulario WAIS y la prueba de vocabulario de imágenes Peabody. Para analizar el efecto de estos indicadores en la prevalencia del DCL se llevó a cabo un análisis de regresión logística. Los resultados indicaron que el grupo con DCL obtuvo puntuaciones significativamente menores que los controles en las tareas de vocabulario y en los hábitos de lectura. Las bajas puntuaciones en las pruebas de vocabulario fueron las medidas de reserva cognitiva que mejor predicen el riesgo de DCL. Teniendo en cuenta los resultados, el buen nivel de vocabulario parece contribuir a aumentar la reserva cognitiva como un indicador protector frente al deterioro cognitivo.

Palabras clave: léxico; memoria; inteligencia cristalizada; deterioro cognitivo; adultos.
\end{abstract}

\section{Introducción}

La reserva cognitiva es un constructo hipotético que hace referencia a la adaptación del cerebro a una situación de deterioro utilizando recursos cognitivos de procesamiento que permiten compensar ese deterioro (Stern, 2007). De esta forma, una persona con alta reserva cognitiva soportará mayor cantidad de neuropatología disminuyendo el riesgo de manifestar los síntomas clínicos de la enfermedad (Andel, Vigen, Mack, Clark, \& Gatz, 2006; De la Barrera, Donolo \& Rinaudo, 2010). Desde un enfoque más pasivo de reserva, otros autores proponen el término de "reserva cerebral" que se basa en aspectos neurobiológicos relacionados con el tamaño cerebral, el número de neuronas o la densidad sináptica. En este sentido, la reserva estaría genéticamente determinada (Carnero-Pardo, 2000).

La reserva cognitiva no se puede medir directamente, por lo que el enfoque de investigación más común es estudiar el efecto de múltiples variables asociadas a la reserva o indicadores, sobre la presencia o no de deterioro cognitivo (Jones et al., 2011). Estos indicadores están relacionados con las experiencias de vida, tales como la educación, la complejidad laboral, la inteligencia cristalizada, las actividades de ocio, el estilo de vida, el estatus socioeconómico... (Rodríguez \& Sánchez, 2004; Stern, 2006).

El nivel de vocabulario es una medida clásica de inteligencia cristalizada que representa el conocimiento verbal y general (Horn \& Cattel, 1967) y, como parte constituyente

* Dirección para correspondencia [Correspondence address]:

Cristina Lojo Seoane. Departamento de Psicoloxía Evolutiva e da Educación, Universidade de Santiago de Compostela. Rúa Xosé María Suárez Núñez, s/n; Campus Vida. 15782 Santiago de Compostela (España). E-mail: cristina.lojo@usc.es
Title: Level of vocabulary as an indicator of cognitive reserve in the evaluation of mild cognitive impairment.

Abstract: This paper studies the role of vocabulary as an indicator of cognitive reserve in the evaluation of mild cognitive impairment (MCI). Three hundred twenty-six participants over 50 years old were classified into two groups, one of 104 patients with MCI and other of 222 healthy controls. We analyzed differences in the scores on different indicators of cognitive reserve, including scores on the WAIS vocabulary subtest and the vocabulary test Peabody. To analyze the effect of these indicators in the prevalence of MCI a logistic regression analysis was carried out. The results indicated that the MCI group had significantly lower scores than the controls in tasks of vocabulary and in reading habits. The low scores on vocabulary tests were the cognitive reserve measures that best predicted the risk of MCI. The high level of vocabulary seems to help the increasing of cognitive reserve as an indicator protective against cognitive decline.

Key words: lexical; memory; crystallized intelligence; cognitive impairment; adults.

del Coeficiente Intelectual, puede ser una poderosa medida de reserva cognitiva (Stern, 2009). Se ha demostrado (Stern et al., 2005) que las personas que poseen alto nivel de vocabulario, medido a través de la puntuación obtenida en el NART y en el WAIS-R, presentan compensación neuronal. Por ello, ha sido utilizado frecuentemente como indicador de reserva cognitiva. Existe una relación bidireccional entre la educación, que es el indicador de reserva cognitiva más estudiado, y la inteligencia, teniendo las personas más inteligentes una mayor tendencia a prolongar su educación y las personas que prolongan su educación, mayor tendencia a desarrollar más su inteligencia (Bilbao-Bilbao \& Seisdedos, 2004). Algunos autores incluso han comprobado que las medidas de inteligencia cristalizada serían un indicador más apropiado de reserva cognitiva y mejor predictor de deterioro que los años de educación, dado que la información sobre los años de educación no siempre es clara ni refleja adecuadamente la habilidad cognitiva de la persona (Alexander et al., 1997; Albert \& Teresi, 1999; Schmand, Smit, Geerlings, \& Lindeboom, 1997). Sumowski, Wylie, DeLuca, y Chiaravalloti (2010) estiman el enriquecimiento intelectual, asociado con una mejor eficiencia cerebral, por la relación entre el nivel educativo, los hábitos de lectura y el vocabulario.

En el proceso de envejecimiento el nivel de vocabulario puede seguir aumentando. Tradicionalmente, se ha medido a través de tests de vocabulario de producción o de respuesta múltiple (Verhaeghen, 2003). En general, en los test de producción empleados, las personas evaluadas deben definir palabras mientras que, en los test de respuesta múltiple, deben seleccionar entre varias alternativas de respuesta que representan un aspecto parcial de la palabra objetivo (dibujos, fotografías, listas de sinónimos, etc.), aquella que más se aproxime a la palabra objetivo. Verhaeghen observó que las diferencias en los efectos de la edad en el desempeño en tests de 
vocabulario variaban significativamente entre formatos de respuesta. Las personas con una media de edad de 70 años rendían mejor en los test de respuesta múltiple que en los tests de producción, comparado con el grupo que tenía una media de edad de 21 años. Bowles y Salthouse (2008) analizaron los efectos de la edad en el rendimiento en pruebas de vocabulario con cuatro formatos de respuesta distintos, dos de ellos de respuesta múltiple (un test de vocabulario de sinónimos y otro de antónimos, en los que el participante debe de elegir una palabra que signifique, respectivamente, lo mismo y lo contrario que la palabra objetivo) y otras dos pruebas de producción (la subescala de vocabulario de WAIS como tarea de definición de palabras y el test de imágenes WJ-R, en el que el participante debe identificar y denominar los objetos representado en fotografías). Estos autores observaron que la relación entre estas cuatro pruebas y la edad fueron no lineales y con un perfil similar de incremento hasta la mediana edad, que los autores estiman en 58 años, y de declive a partir de ese punto. De forma consistente con los resultados de Verhaeghen (2003), este declive es mayor para los test de producción que para los test de respuesta múltiple. Teniendo en cuenta los resultados observados en el rendimiento en las pruebas de vocabulario, parece que el nivel de vocabulario aumenta hasta una determinada edad a partir de la cual, puede experimentar un declive. Este declive parece ser más evidente en pruebas de vocabulario de producción.

Juncos-Rabadán, Pereiro, Facal, y Rodríguez (2010) realizaron una revisión sobre el vocabulario y el acceso al léxico en los estudios poblacionales y experimentales sobre Deterioro Cognitivo Ligero (DCL), entendido como el estadio intermedio situado en un continuo entre el envejecimiento normal y la Enfermedad de Alzheimer (EA) (Petersen et al., 1999). En los estudios poblacionales, tan solo se encontró un estudio que contempla las bajas puntuaciones en vocabulario como un predictor de DCL y de su conversión a EA (Holtzer, Verghese, Wang, Hall, \& Lipton, 2008). En los estudios experimentales, se ha analizado la relación del nivel de vocabulario en el DCL con los cambios en la capacidad de acceso al léxico, medidos a través del paradigma experimental del fenómeno punta de la lengua (Juncos-Rabadán, Facal, Rodríguez, \& Pereiro, 2010; Rodríguez, Juncos-Rabadán, \& Facal, 2008). Se ha encontrado que el mayor nivel de vocabulario en el DCL es un factor protector que contribuye a mejorar el acceso a los nombres comunes pero que no tiene efecto en el acceso a los nombres propios (Juncos-Rabadán, Rodríguez, Facal, Cuba, \& Pereiro, 2011).

Contemplando la medida de vocabulario como indicador de reserva cognitiva, el nivel de vocabulario tendría un efecto protector frente a la aparición y desarrollo del DCL (Lojo-Seoane, Facal, \& Juncos-Rabadán, 2012). Solé-Padullés y cols. (2009) estudiaron la relación entre distintos indicadores de reserva cognitiva, la actividad cerebral y las características estructurales cerebrales en 44 participantes mayores de 65 años, de los cuales 16 eran controles, 12 DCL-amnésicos y 16 con EA leve. Se observaron correlaciones negativas entre el volumen cerebral y la puntuación en el subtest de vocabulario WAIS en los grupos de DCL y EA. Estos autores explican que los participantes con mayor puntuación en el WAIS muestran un proceso neuropatológico mayor reflejado en la mayor atrofia de sus cerebros en relación a su volumen cerebral. Apoyan la hipótesis del umbral según la cual los individuos con mayor reserva permanecen clínicamente sanos a pesar de su avanzada atrofia cerebral, pero cuando aparecen manifestaciones clínicas, como en el DCL, su declive puede ser mayor que el de las personas con menor reserva. Además, observaron un incremento del volumen de la materia gris en el cerebro de las personas con mayor puntuación en las medidas del WAIS incluido el subtest de vocabulario, confirmando los hallazgos previos de Colom, Jung, y Haier (2006). Fritsch y cols. (2005) combinaron el efecto de los indicadores de reserva de actividad cognitiva y la inteligencia, relacionando el rendimiento y la actividad cognitiva en la juventud con la presencia de deterioro cognitivo en la vejez. Los resultados indicaron que un alto CI y una mayor actividad cognitiva en la adolescencia fueron asociados con un menor riesgo de deterioro, siendo el riesgo de deterioro la mitad para las personas que se desvían un punto por encima de la media de inteligencia que para las personas que están en la media. Además, también encontraron menor riesgo de deterioro para las personas que participaron en dos o más actividades por año, frente a las que participaron en menos.

El objetivo del presente trabajo es profundizar en la relación del nivel de vocabulario con el DCL, analizando su valor predictivo sobre las manifestaciones clínicas del DCL. Para ello, se analizan las diferencias entre las puntuaciones obtenidas por los grupos DCL y control en los distintos indicadores de reserva cognitiva, incluyendo las medidas de vocabulario, así como la relación entre los indicadores de reserva y la prevalencia de deterioro cognitivo.

\section{Método}

\section{Participantes}

Participaron un total de 326 personas mayores de 50 años que presentaban quejas subjetivas de memoria, 218 hombres y 108 mujeres, que acudían a centros de salud de las ciudades de Santiago de Compostela y Vigo y que eran derivados por sus médicos de familia para que se le realizara una valoración cognitiva en sus propios centros por parte de psicólogos especializados. Para la derivación de los participantes, se consideraron los siguientes criterios de exclusión: diagnóstico previo de demencia o cualquier tipo de enfermedad neurodegenerativa, deficiencia mental, alteraciones psiquiátricas y/o enfermedad sistémica grave (hepática, renal, etc.), y alteraciones perceptivas y motrices que impidieran la normal aplicación de las pruebas. Los participantes fueron asignados a dos grupos: DCL $(n=104)$ y Control $(n$ $=222$ ). En el grupo de DCL se incluyeron pacientes con DCL amnésico unidominio o multidominio, siguiendo los 
criterios de Petersen y cols. (1999): 1) quejas subjetivas de memoria confirmadas por un informante; 2) rendimiento cognitivo general dentro de la normalidad (para los unidominio), y tener una puntuación 1.5 veces la desviación estándar por debajo de la media de su grupo normativo en el MMSE según las normas de Lobo y cols. (1999), y al menos en dos escalas del CAMCOG-R (Roth, Huppert, Mountjoy, \& Tym, 2003) distintas de la memoria para los multidominio; 3) puntuaciones 1.5 veces la desviación estándar por debajo de la media de su grupo normativo en memoria libre a corto plazo del test TAVEC (Benedet \& Alejandre, 1998); 4) no tener alteraciones en las actividades de la vida diaria; y 5 ) no cumplir los criterios de demencia. El grupo control lo forman personas sanas sin ningún tipo de deterioro cognitivo que podían presentar quejas subjetivas de memoria. En la Tabla 1 se describen las características de la muestra seleccionada por grupos en cuanto a edad media, sexo y puntuaciones medias en el MMSE y en recuerdo libre a corto plazo (rlcp) del TAVEC.

Tabla 1. Características de la muestra. Medias y Desviaciones típicas (entre paréntesis) en edad, MMSE y TAVEC-rlcp (recuerdo libre a corto plazo)

\begin{tabular}{|c|c|c|c|c|c|c|}
\hline \multirow[t]{2}{*}{ GRUPO } & \multirow[t]{2}{*}{$n$} & \multirow[t]{2}{*}{ Edad } & \multicolumn{2}{|c|}{ Sexo } & \multirow[t]{2}{*}{ MMSE } & \multirow[t]{2}{*}{ TAVEC- rlcp } \\
\hline & & & Hombres & Mujeres & & \\
\hline DCL multidominio amnésico & 44 & $\begin{array}{l}70.27 \\
(9.51)\end{array}$ & 14 & 30 & $\begin{array}{l}22.95 \\
(1.66)\end{array}$ & $\begin{array}{c}3.45 \\
(2.15)\end{array}$ \\
\hline DCL multidominio no amnésico & 25 & $\begin{array}{l}65.28 \\
(8.70)\end{array}$ & 2 & 23 & $\begin{array}{l}22.88 \\
(1.51)\end{array}$ & $\begin{array}{c}9.56 \\
(2.08)\end{array}$ \\
\hline DCL unidominio amnésico & 35 & $\begin{array}{l}67.54 \\
(9.27)\end{array}$ & 17 & 18 & $\begin{array}{l}26.77 \\
(1.93)\end{array}$ & $\begin{array}{c}4.11 \\
(2.23)\end{array}$ \\
\hline CONTROL & 222 & $\begin{array}{l}65.9 \\
(8.8) \\
\end{array}$ & 75 & 147 & $\begin{array}{l}27.32 \\
(1.93)\end{array}$ & $\begin{array}{l}10.08 \\
(2.73)\end{array}$ \\
\hline
\end{tabular}

\section{Materiales y procedimiento}

Para la valoración de la reserva cognitiva se utilizaron medidas recogidas en los datos sociodemográficos de la entrevista realizada como: años de escolarización, profesión, hábitos de lectura, actividades sociales y actividades culturales. Además, como medidas de inteligencia cristalizada, se utilizó la puntuación obtenida por los participantes en dos pruebas de vocabulario: el subtest de vocabulario de la Escala de Inteligencia de Wechsler para adultos (Wechsler, 1988) y la prueba de vocabulario de imágenes Peabody, adaptación al castellano del Peabody Picture Vocabulary Test- Revised (Dunn \& Dunn, 1981). El subtest de vocabulario WAIS (Wechsler, 1988) está compuesto por 40 palabras que el investigador debe ir leyendo para que el participante proporcione una definición para ellas. Es un test de producción, que mide la capacidad de expresar y explicar el significado de palabras. Se puntúa con 2 puntos las definiciones acertadas y abstractas; con 1 punto las definiciones descriptivas, concretas, funcionales o vagas; con 0 puntos las definiciones en las que el participante no muestra una comprensión razonable de la palabra o bien la ausencia de definición de la misma. Por su parte, en el test de vocabulario de imágenes (Dunn \& Dunn, 1981) se aplican 100 láminas precedidas por 3 ítems de práctica. En cada lámina se presentan cuatro ilustraciones, sencillas, en blanco y negro, organizadas en forma de selección múltiple (numeradas de 1 al 4). Se trata de un test de respuesta múltiple, en el que el participante selecciona la lámina (diciendo su número en voz alta) que mejor ilustra el significado de la palabra que el examinador le presenta oralmente.

Los materiales indicados fueron administrados en los propios centros de salud dentro de un protocolo general de evaluación realizada por psicólogos/as debidamente entre- nados. El estudio forma parte de un proyecto de investigación más amplio y contaba con la aprobación del Comité Ético de Investigaciones Clínicas de Galicia y fue posible su realización gracias al apoyo del Servizo Galego de Saúde (SERGAS). Todos los participantes debían firmar un consentimiento informado autorizando su participación voluntaria en el estudio.

\section{Análisis estadístico}

Para explorar las diferencias entre las puntuaciones de los grupos en los indicadores de reserva cognitiva se hicieron ANCOVAs incluyendo la edad como covariable. En los casos en los que se incumplía el supuesto de homogeneidad de varianzas se aplicó la prueba U de Mann Whitney.

Con el objetivo de analizar cómo se relacionan los distintos indicadores de reserva cognitiva, se ha realizado un análisis de correlaciones, calculándose las correlaciones bivariadas de Pearson para las variables cuantitativas discretas y correlaciones bivariadas de Spearman para las variables de escala ordinales (variables de reserva medidas como frecuencias: complejidad laboral, frecuencia de lectura, actividades sociales y actividades culturales).

Asimismo, se realizó un análisis de regresión logística para evaluar la relación entre el bajo nivel de reserva cognitiva medido a través de sus indicadores y el DCL. Este método nos permite introducir variables predictoras categóricas y cuantitativas y obtener la Odds Ratio $(O R)$ de cada una de ellas que se corresponde con el riesgo de presentar DCL.

Las variables utilizadas como indicadores de reserva son las siguientes: A) Los años de escolarización, que para el análisis de regresión se han clasificado en cuatro niveles de acuerdo con la categorización normativa de la versión de Lobo y colaboradores del MMSE: estudios básicos (0-4 
años), estudios primarios (5-8 años), estudios secundarios (912 años) y estudios universitarios (+12). B) La complejidad laboral, clasificada inicialmente teniendo en cuenta los criterios del NEST-DD (Garibotto et al., 2008), se ha transformado en una variable dicotómica como alta y baja complejidad laboral. C) Las frecuencias de participación en hábitos de lectura, actividades sociales y actividades culturales se han transformado en variables dicotómicas clasificándolas como frecuentes o infrecuentes. Como medidas de vocabulario se han utilizado las puntuaciones de los participantes en los test Wechsler y Peabody y se han clasificado en cuatro categorías en función de los percentiles 25, 50 y 75 obtenidos en la muestra de participantes del grupo control.

\section{Resultados}

Se realizaron comparaciones entre las distintas puntuaciones obtenidas por los participantes de los grupos DCL y control en los distintos indicadores de reserva cognitiva (ANCOVA y U de Mann Whitney). Los resultados se presentan en la Tabla 2. Se han encontrado diferencias significativas en la edad, siendo la edad media del grupo de participantes con DCL mayor que la del grupo control $(F(1,324)=4.45 ; p=$ .036). Asimismo, se observan diferencias significativas en las puntuaciones medias obtenidas por los participantes en tres de las medidas de reserva cognitiva: hábitos de lectura $(Z=-$ 2.03; $p=.043)$, puntuaciones en vocabulario WAIS $(F(1,323)=15.97 ; p<.0001)$ y puntuaciones de Peabody $(F(1,323)=8.65 ; p=.004)$. En estos indicadores, tanto el efecto de la covariable edad como efecto principal de grupo resultan significativos. En el caso de las puntuaciones obtenidas en el indicador de años de escolarización se observó un efecto significativo de la covariable edad que anula el efecto de grupo. En los indicadores de complejidad laboral y actividades sociales y culturales no se encontraron diferencias significativas.

Tabla 2. Medias (con desviaciones estándar entre paréntesis) y diferencias entre el grupo control y DCL en los indicadores de reserva cognitiva

\begin{tabular}{|c|c|c|c|c|}
\hline & DCL & Control & Total & Diferencias intergrupo \\
\hline$\overline{\text { Edad }}$ & $\begin{array}{l}68.15 \\
(9.37)\end{array}$ & $\begin{array}{l}65.90 \\
(8.80)\end{array}$ & $\begin{array}{l}66.62 \\
(9.03)\end{array}$ & $F(1,324)=4.45^{a^{*}}$ \\
\hline Años de escolarización & $\begin{array}{l}9.13 \\
(4.15)\end{array}$ & $\begin{array}{c}9.61 \\
(4.57)\end{array}$ & $\begin{array}{l}9.45 \\
(4.44)\end{array}$ & $F(1,323)=0.43^{\mathrm{b}}$ \\
\hline Complejidad laboral & $\begin{array}{l}3.06 \\
(1.04)\end{array}$ & $\begin{array}{c}3.14 \\
(1.07)\end{array}$ & $\begin{array}{c}3.12 \\
(1.06)\end{array}$ & $F(1,323)=0.28^{b}$ \\
\hline Hábitos de lectura & $\begin{array}{c}2.97 \\
(1.23)\end{array}$ & $\begin{array}{l}3.29 \\
(1.05)\end{array}$ & $\begin{array}{l}3.19 \\
(1.12)\end{array}$ & $Z=-2.03^{\mathrm{c}^{*}}$ \\
\hline Participación social & $\begin{array}{l}2.27 \\
(1.2)\end{array}$ & $\begin{array}{c}2.2 \\
(1.1)\end{array}$ & $\begin{array}{c}2.22 \\
(1.13)\end{array}$ & $Z=-2.87^{c}$ \\
\hline Participación cultural & $\begin{array}{l}1.72 \\
(1.19)\end{array}$ & $\begin{array}{c}1.64 \\
(1.09)\end{array}$ & $\begin{array}{c}1.67 \\
(1.12)\end{array}$ & $F(1,323)=0.20^{\mathrm{b}}$ \\
\hline Puntuación vocabulario Peabody & $\begin{array}{c}55.37 \\
(16.89)\end{array}$ & $\begin{array}{c}62.12 \\
(16.92)\end{array}$ & $\begin{array}{c}59.96 \\
(17.18)\end{array}$ & $F(1,323)=8.65^{b^{* *}}$ \\
\hline $\begin{array}{l}\text { Puntuación vocabulario } \\
\text { WAIS }\end{array}$ & $\begin{array}{c}42.42 \\
(12.04)\end{array}$ & $\begin{array}{c}49.22 \\
(13.10)\end{array}$ & $\begin{array}{c}47.05 \\
(13.44)\end{array}$ & $F(1,323)=15.97 \mathrm{~b}^{* *}$ \\
\hline
\end{tabular}

a Anova (valor F)

b Ancova con edad como covariable (valor F)

c. U Mann Whitney (valor Z)

${ }^{*} p<.05 ;{ }^{* *} p<.01$

Los resultados de los análisis de correlaciones entre los distintos indicadores de reserva cognitiva se presentan en la Tabla 3. En ella se observa que existe una relación significativa entre los indicadores de años de escolarización, complejidad laboral, hábitos de lectura y las medidas de vocabulario. Todos estos indicadores se relacionan significativamente en- tre sí. En el caso de la puntuación en WAIS también existe una correlación significativa con la participación en actividades culturales. La participación en actividades sociales correlaciona significativamente con la participación en actividades culturales.

Tabla 3. Matriz de correlaciones entre las variables de reserva cognitiva

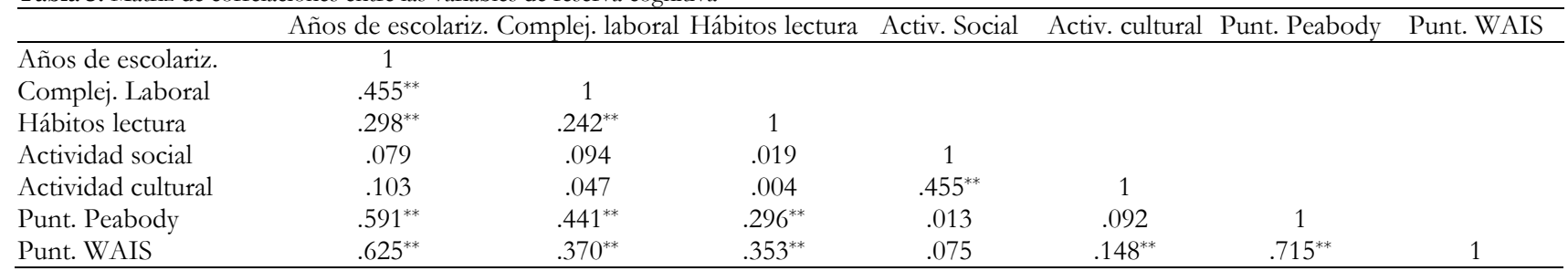


Los resultados de la regresión logística se sintetizan en la Tabla 4. En ella, se puede observar que la prevalencia de DCL es mayor en los participantes con hábitos de lectura infrecuentes que en los que tienen hábitos de lectura frecuentes $(O R=2.17 ; 95 \% C I=1.29-3.65)$. Los hábitos de lectura infrecuentes explican un $2.6 \%$ de la varianza del DCL.

Respecto a las medidas de vocabulario, las bajas puntuaciones tanto en la prueba de vocabulario Peabody como en el WAIS han demostrado ser predictoras de DCL. Así, se observa una mayor prevalencia de DCL en el grupo de participantes que obtienen una menor puntuación en el test de vocabulario Peabody, que en los participantes que obtienen mayor puntuación (puntuación mayor a 77) $(O R=4.51 ; 95 \%$ $C I=1.87-10.91)$. Una puntuación baja en esta prueba explica un $4.9 \%$ de la varianza del DCL. De igual forma, también se obtiene mayor prevalencia de DCL en los participantes que obtienen una puntuación entre 57 y 65 que en los participantes que obtienen puntuaciones mayores de $77(\mathrm{OR}=$ 3.34; $95 \% C I=1.34-8.31)$. Sin embargo, no existen diferencias significativas en cuanto a la prevalencia de DCL entre los que obtienen una puntuación entre 66-77 y los que obtienen puntuaciones mayores a 77 .
En cuanto a la escala WAIS de vocabulario, los resultado son similares a los obtenidos en el test de vocabulario Peabody. Existe una mayor prevalencia de DCL en los participantes que obtienen una menor puntuación en la escala (puntuación de 0 a 42, por debajo del percentil 25), que en los que obtienen la mayor puntuación (puntuación mayor que 62, por encima del percentil 75) $(O R=4.28 ; 95 \% C I=$ 1.86-9.87). Una puntuación baja en la prueba de vocabulario WAIS explica un $5.5 \%$ de la varianza del DCL. También se observa mayor prevalencia de DCL en los participantes que obtienen una puntuación entre 43 y 52 (debajo del percentil 25) que en los que obtienen una puntuación mayor que 62 $(O R=3.01 ; 95 \% C I=1.26-7.22)$. Sin embargo, no existen diferencias significativas entre los que obtienen una puntuación entre 53-62 (entre percentil 50 y 75), y aquellos cuyas puntuaciones son mayores que 62 (por encima del percentil 75).

No existen diferencias significativas en la prevalencia de DCL asociado a los indicadores de años de escolarización, complejidad laboral y frecuencia de participación en actividades sociales y culturales.

Tabla 4. Prevalencia de deterioro cognitivo asociada a los distintos indicadores de reserva cognitiva (Regresión logística bivariada)

\begin{tabular}{|c|c|c|c|c|c|c|c|}
\hline Indicadores de reserva & & Casos & $\%$ & $\chi^{2}$ Wald & Valores $p$ & OR & $95 \% C I$ \\
\hline \multirow[t]{4}{*}{ Años de educación } & $0-4$ & $9 / 26$ & 34.61 & 2.05 & .44 & 1.46 & $0.56-3.79$ \\
\hline & $5-8$ & $46 / 147$ & 31.29 & 0.59 & .48 & 1.25 & $0.67-2.33$ \\
\hline & $9-12$ & $29 / 78$ & 37.18 & 0.51 & .17 & 1.63 & $0.82-3.24$ \\
\hline & $13+$ & $20 / 75$ & 26.67 & & & & \\
\hline \multirow[t]{2}{*}{ Complejidad laboral } & Baja & $64 / 190$ & 33.68 & 0.67 & .42 & 1.22 & $0.76-1.96$ \\
\hline & Alta & $40 / 136$ & 29.41 & & & 1 & \\
\hline \multirow[t]{2}{*}{ Hábitos de lectura } & Infrecuentes & $37 / 82$ & 45.12 & 8.62 & .003 & 2.17 & $1.29-3.65$ \\
\hline & Frecuentes & $67 / 244$ & 27.46 & & & 1 & \\
\hline \multirow[t]{2}{*}{ Actividad social } & Infrecuentes & $58 / 199$ & 29.15 & 1.78 & .18 & 0.72 & $0.45-1.16$ \\
\hline & Frecuentes & $46 / 127$ & 36.22 & & & 1 & \\
\hline \multirow[t]{2}{*}{ Actividad cultural } & Infrecuentes & $78 / 253$ & 30.83 & 0.6 & .44 & 0.81 & $0.47-1.39$ \\
\hline & Frecuentes & $26 / 73$ & 35.62 & & & 1 & \\
\hline \multirow[t]{4}{*}{ Puntuación Peabody } & $0-56$ & $47 / 111$ & 42.34 & 11.18 & .001 & 4.51 & $1.87-10.91$ \\
\hline & $57-65$ & $31 / 88$ & 35.23 & 6.74 & .009 & 3.34 & $1.34-8.31$ \\
\hline & $66-77$ & $19 / 77$ & 24.67 & 2.07 & .15 & 2.01 & $0.78-5.21$ \\
\hline & $78+$ & $7 / 50$ & 14 & & & 1 & \\
\hline \multirow[t]{4}{*}{ Puntuación WAIS } & $0-42$ & $51 / 118$ & 43.22 & 11.64 & .001 & 4.28 & $1.86-9.87$ \\
\hline & $43-52$ & $30 / 86$ & 34.88 & 6.13 & .013 & 3.01 & $1.26-7.22$ \\
\hline & $53-61$ & $15 / 69$ & 21.74 & 0.86 & .36 & 1.56 & $0.61-4.02$ \\
\hline & $62+$ & $8 / 53$ & 15.09 & & & 1 & \\
\hline
\end{tabular}

\section{Discusión}

Los análisis de diferencias entre el grupo DCL y control en los distintos indicadores de reserva cognitiva revelan que existen diferencias significativas entre los dos grupos en los hábitos de lectura y en las puntuaciones en vocabulario, tanto en la prueba de vocabulario de imágenes Peabody como en el subtest de vocabulario WAIS. El hecho de que resultaran significativas en la diferenciación de DCL las medidas de vocabulario y de hábitos de lectura de entre todos los indicadores de reserva cognitiva, sugiere que podría existir alguna relación entre la inteligencia y los hábitos de lectura, tal y como planteaban Sumowski y cols (2010), los cuales las consideraban como medidas de enriquecimiento intelectual. En 
cuanto al nivel educativo, que Sumowski y cols (2010) también incluían como medida de enriquecimiento intelectual, en este estudio no se encuentran diferencias significativas entre los participantes del grupo control y DCL debido al efecto de la edad, pues la mayor edad se asocia en esta muestra de personas mayores con un menor nivel educativo.

En los resultados obtenidos en el análisis de regresión logística se observa, teniendo en cuenta la proporción de varianza del deterioro explicada de cada uno de los indicadores analizados y el valor de $O R$, que el riesgo de presentar DCL es mayor si se obtienen puntuaciones bajas en los test Peabody y WAIS que si los hábitos de lectura son infrecuentes. Este hallazgo concuerda con los obtenidos por Holtzer y cols. (2008), los cuáles observaron que el subtest de vocabulario WAIS discriminaba a los participantes con DCL y, además, sugería, que sus puntuaciones podrían ser útiles para predecir la demencia.

Nuestros resultados también coinciden con los de Fritsch y cols. (2005) que observaron que existía la mitad de riesgo de deterioro para las personas que se desvían un punto por encima de la media de inteligencia que para las personas que están en la media. Los resultados presentados también muestran que el menor nivel de vocabulario, con puntuaciones por debajo del percentil 50, reflejo de una reducida inteligencia cristalizada, implica un riesgo de DCL entre tres y cuatro veces mayor que los que tienen puntuaciones superiores al percentil 75. También coinciden con los de Fritsch y colaboradores los resultados sobre el efecto de la frecuencia de los hábitos de lectura en el DCL, observando que son las personas con hábitos de lectura infrecuentes las que tienen mayor riesgo de presentar DCL.

En esta misma línea nuestros resultados sobre el vocabulario como una medida de inteligencia cristalizada apoyan el planteamiento de diversos autores sobre el papel protector de la inteligencia frente al declive cognitivo y la evolución del proceso neuropatológico (Schmand et al., 1997; SoléPadullés et al., 2009).

Nuestros resultados indican que un menor nivel de vocabulario predice la prevalencia de DCL. Esto corroboraría el efecto protector del vocabulario como indicador de reserva cognitiva que facilitaría que el cerebro disponga de más recursos para compensar y, por lo tanto, retrasaría la manifestación clínica de un posible deterioro cognitivo. Además, el análisis de correlaciones presentado indica que las personas con alto nivel de vocabulario presentan altas puntuaciones en otros indicadores de reserva cognitiva con los que está relacionado, como los hábitos de lectura, los años de esco-

\section{Referencias}

Albert, S. M., \&, Teresi, J. A. (1999). Reading ability, education, and cognitive status assessment among older adults in Harlem. American Journal of Public Health, 89(1), 95-97. Doi:10.2105/AJPH.89.1.95

Alexander, G. E., Furey, M. L., Grady, C. L., Pietrini, P., Brady, D. R., Mentis, M. J., \& Schapiro, M.B. (1997). Association of premorbid function with cerebral metabolism in Alzheimer's disease: implications for the reserve hypothesis. American Journal Psychiatric, 154(2), 165-172. larización o la complejidad laboral. Una mayor reserva cognitiva no afectaría tan solo al retraso de la aparición de los síntomas de DCL, sino también, a su desarrollo (LojoSeoane et al., 2012). De esta manera, una persona con bajo nivel de vocabulario y menor reserva cognitiva manifestará más pronto los síntomas en el proceso de deterioro y presentará un desarrollo gradual de los mismos. Sin embargo una persona con alto nivel de vocabulario y mayor reserva cognitiva se mantendrá por más tiempo en el proceso de deterioro sin manifestar los síntomas clínicos de la enfermedad hasta llegar a un punto en el proceso de deterioro en el que no es posible que compense más el daño ocasionado por la patología. Como consecuencia, la manifestación del deterioro de una persona con alta reserva cognitiva se podría producir más tarde en el proceso de deterioro pero de forma más acusada. Estos resultados que demuestran la influencia del nivel de vocabulario, medido a través del subtest de vocabulario WAIS, en el DCL, corroboran los resultados observados por Colom y cols. (2006) y Solé-Padullés y cols. (2009).

Por lo tanto, el vocabulario refleja un conocimiento cristalizado que se mantiene con el paso de los años en un envejecimiento normal y que actuaría como enmascarador de la manifestación de los síntomas de deterioro, aumentando la reserva cognitiva de las personas. Además, el declive en las puntuaciones obtenidas en las pruebas que lo valoran permiten diferenciar a personas con DCL de las personas sin deterioro cognitivo, por lo que podría ayudar a predecir una posible conversión a demencia. Esperamos que el estudio longitudinal que está llevando a cabo nuestro equipo de investigación pueda aportar evidencias con respecto a esas hipótesis. Estos hallazgos pueden aportar información relevante para el diagnóstico y medición del DCL, tanto por su efecto enmascarador de los síntomas clínicos del deterioro como por su efecto discriminador del mismo. Además aporta información para el desarrollo de intervenciones cognitivas que tienen como objetivo el retraso del deterioro cognitivo y en las que, teniendo en cuenta los resultados de este estudio, convendría incluir un entrenamiento que facilite el aumento del nivel de vocabulario.

Agradecimientos.- Este trabajo forma parte del proyecto de investigación "Estudio longitudinal sobre marcadores cognitivos en el envejecimiento cognitivo normal, el deterioro cognitivo ligero y la enfermedad de Alzheimer" financiado por el Ministerio de Ciencia e Innovación (Ref. PSI2010-22224-C03-01).

Andel, R., Vigen, C., Mack, W. J., Clark, L. J., \& Gatz, M. (2006). The effect of education and occupational complexity on rate of cognitive decline in Alzheimer's patients. Journal of the International Neuropsychological Society, 12(1), 147-152. Doi:10.1017/S1355617706060206

Benedet, M. J., \& Alejandre, M. A. (1998). TAVEC: Test de aprendizaje verbal España-complutense. Madrid: TEA Ediciones. 
Bilbao-Bilbao, A., \& Seisdedos, N. (2004). Eficacia de una fórmula de estimación de la inteligencia premórbida en la población española. Revista de Neurología, 38(5), 431-434.

Bowles, R. P., \& Salthouse, T. A. (2008). Vocabulary test format and differential relations to age. Psychology and Aging, 23(2), 366-376. Doi: 10.1037/0882-7974.23.2.366

Carnero-Pardo, C. (2000). Educación, demencia y reserva cerebral. Revista de Neurología, 31(6), 584-592.

Colom, R., Jung, R. E. y Haier, R. J. (2006). Distributed brain sites for the gfactor intelligence. Neuroimage, 31, 1359-1365. Doi:10.1016/j.neuroimage.2006.01.006

De la Barrera, M. L., Donolo, D., \& Rinaudo, M. C. (2010). Riesgo de demencia y niveles de educación: Cuando aprender es más saludable de lo que pensamos. Anales de psicología, 26(1), 34-40. Doi:10.6018/analesps.26.1.91941

Dunn, L. M., \& Dunn, L. M. (1981). Peabody picture vocabulary test- revised. Circle Pines: American Guidance Service.

Fritsch, T., Smyth, K., McClendon, M., Ogrocki, P., Santillan, C., Larsen, J. ...Strauss, M. (2005). Associations between Dementia/Mild cognitive impairment and cognitive performance and activity levels in youth. Journal of the American Geriatrics Society, 53(7), 1191-1196. Doi:10.1111/j.1532-5415.2005.53361.x

Garibotto, V., Borroni, B., Kalbe, E., Herholz, K., Salmon, E., Holtoff, V. ...Perani, D. (2008). Education and occupation as proxies for reserve in aMCI converters and AD FDG-PET evidence. Neurology, 71(17), 13421349. Doi:10.1212/01.wnl.0000327670.62378.c0

Holtzer, R., Verghese, J., Wang, C., Hall, C. B., \& Lipton, R. B. (2008). Within-Person Across Neuropsychological Test Variability and Incident Dementia. JAMA 300(7), 823-830. Doi:10.1001/jama.300.7.823

Horn, J. L., \& Cattell, R. B. (1967). Age differences in fluid and crystallized intelligence. Acta Psychologica, 26, 107-129. Doi:10.1016/00016918(67)90011-X

Jones, R. H., Manly, J., Glymour, M. M., Rentz, D. M., Jefferson, A. L., \& Stern, Y. (2011). Conceptual and measurement challenges in research on cognitive reserve. Journal of the International Neuropsychological Society, 17, 1-9. Doi:10.1017/S1355617710001748

Juncos-Rabadán, O., Facal, D., Rodríguez, M.S., \& Pereiro, A. X. (2010). Lexical knowledge and lexical retrieval in aging. Insights from a Tip-ofthe-tongue (TOT) study. Language and Cognitive Processes, 25, 1301-1334. Doi:10.1080/01690961003589484

Juncos-Rabadán, O., Rodríguez, M. S., Facal, D., Cuba, J., \& Pereiro, A. X. (2011). Tip-of-the-tongue for proper names in mild cognitive impairment. Semantic or post-semantic impairments? Journal of Neurolinguistics, 24(6), 636-651. Doi:10.1016/j.jneuroling.2011.06.004

Juncos-Rabadán, O., Pereiro, A.X., Facal, D., \& Rodríguez, N. (2010). Una revisión de la investigación sobre lenguaje en el deterioro cognitivo leve. Revista de Logopedia, Foniatría y Audiología, 30(2), 73-83. Doi:10.1016/S0214-4603(10)70119-4

Lobo, A., Saz, P., Marcos, G., Día, J. L., de la Cámara, C., Ventura, T., ... Aznar, S. (1999). Revalidación y normalización del mini- examen cognoscitivo (primera versión en castellano del Mini-Mental Status Exami- nation) en la población general geriátrica. Medicina Clínica, 112(20), 767774.

Lojo-Seoane, C., Facal, D., \& Juncos-Rabadán, O. (2012) ¿Previene la actividad intelectual el deterioro cognitivo? Relaciones entre reserva cognitiva y deterioro cognitivo ligero. Revista Española de Geriatría y Gerontología, 47(6), 270-278. Doi:10.1016/j.regg.2012.02.006

Petersen, R. C., Smith, G. E., Waring, S. C., Ivnik, R. J., Tangalos, E. G., \& Kokmmen, E. (1999). Mild cognitive impairment: Clinical characterization and outcome. Archives of Neurology, 56, 303-308. Doi:10.1001/archneur.56.3.303

Rodríguez, N., Juncos-Rabadán, O., \& Facal, D. (2008). El fenómeno de la punta de la lengua en el deterioro cognitivo leve. Un estudio piloto. Revista de Logopedia, Foniatría y Audiología, 28(1), 28-33. Doi:10.1016/S02144603(08)70043-3

Rodríguez, M., \& Sánchez, J. L. (2004). Reserva cognitiva y demencia/Cognitive reserve and dementia. Anales de Psicología. Special Issue: Current Research Lines in Neuropsychology, 20(2), 175-186.

Roth, M., Huppert, F. A., Mountjoy, C. Q., \& Tym, E. (2003) Prueba de exploración Cambridge revisada para la valoración de los trastornos mentales en la vejez. Madrid: TEA.

Schmand, B., Smit, J. H., Geerlings, M. I., \& Lindeboom, J. (1997). The effects of intelligence and education on the development of dementia: A test of the brain reserve hypothesis. Psychological Medicine: A Journal of Research in Psychiatry and the Allied Sciences, 27(6), 1337-1344. Doi:10.1017/S0033291797005461

Solé-Padullés, C., Bartrés-Faz, D., Junqué, C., Vendrell, P., Rami, L., Clemente, I. C. ... Molinuevo, J. L. (2009). Brain structure and function related to cognitive reserve variables in normal aging, mild cognitive impairment and Alzheimer's disease. Neurobiology of Aging, 30(7), 11141124. Doi:10.1016/j.neurobiolaging.2007.10.008

Stern, Y. (2006). Cognitive reserve and Alzheimer disease. Alzheimer Disease and Associated Disorders, 20(2), 112-117. Doi:10.1097/00002093200607001-00010

Stern, Y. (2007). Cognitive reserve. Theory and applications. New York: Taylor \& Francis.

Stern, Y. (2009). Cognitive reserve. Neuropsychologia 47, 2015-2028. Doi: http://dx.doi.org/10.1016/j.neuropsychologia.2009.03.004

Stern, Y., Habeck, C., Moeller, J., Scarmeas, N., Anderson, K. E., Hilton, H. J. ... van Heertum, R., (2005). Brain networks associated with cognitive reserve in healthy young and old adults. Cerebral Cortex, 15, 394-402.

Sumowski, J. F., Wylie, G. R., DeLuca, J., \& Chiaravalloti, N. (2010). Intellectual enrichment is linked to cerebral efficiency in multiple sclerosis: Functional magnetic resonance imaging evidence for cognitive reserve. Brain: A Journal of Neurology, 133(2), 362-374. Doi:10.1093/brain/awp307

Verhaeghen, P. (2003). Aging and vocabulary scores: a metaanalysis. Psychology and Aging, 18, 332-339. Doi: 10.1037/0882-7974.18.2.332

Wechsler, D. (Ed.). (1988). WAIS-R: Wechsler adult intelligence scale- revised. Oxford: Psychological Corporation.

(Articulo recibido: 20-9-2012; revisión recibida: 3-3-2013; aceptado: 12-3-2013) 